Biotempo (Lima)

ORIGINAL ARTICLE / ARTÍCULO ORIGINAL

RESTRICTIONS OF THE LIQUOR EXPENDED SCHEDULES IN LIMA METROPOLITANA, PERU AND ITS EFFECT ON THE VIOLENT DEATH 2015-2017

\title{
RESTRICCIONES DE LOS HORARIOS DE EXPENDIO DE LICORES EN LIMA METROPOLITANA, PERU Y SU EFECTO EN LAS MUERTES VIOLENTAS 2015-2017
}

\author{
Hernan Malaga ${ }^{1}$; Juan Ronceros ${ }^{2}$; Luis Delgado ${ }^{1} \&$ Gerardo Palza $^{3}$
}

\author{
Universidad Ricardo Palma. Lima, Perú. \\ Municipalidad de Miraflores. Lima, Perú. \\ Instituto de Medicina Legal del Perú. \\ Author for correspondence: hernan.malaga@urp.edu.pe
}

\begin{abstract}
The objective of the present investigation was to determine the relationship between the "Safe Hour" liquor sales schedules and those killed by traffic crashes and abuses, homicides and suicides. The descriptive investigation based on the monthly reports carried out by the Metropolitan Municipality of Lima (LM), Peru from the database of the Institute of Legal Medicine (ILM) recorded from 2015 to 2017, with a bivariate analysis and relative risks, characterizing those killed by violent deaths and their relationship with sex, age and alcohol consumption. There is a significant decrease in alcoholized victims in all violent deaths except for the suicide of women, between 2015 and 2017. The largest percentage decrease in homicides of alcoholics occurred in women, meaning a reduction in feminicide. The average age shifts in frequencies as the years go by, with the exception of suicide in women between the base year (2015) against 16 and 17 . The victims are older in LM alcoholics, consistent with that published by the statistical yearbook of the National Police, both in age and sex. It is concluded that the decrease in violent deaths and changes in age and sex in LM should be a consequence of public policy and effective police controls. The "safe hour" has evidence for its application throughout the country. Safe time is an effective strategy to deal with feminicide.
\end{abstract}

Keywords: abuses - homicides - restriction of liquor sales schedules - suicides - traffic crash - violent deaths

\section{RESUMEN}

El objetivo de la presente investigación fue determinar la relación entre los Horarios de expendio de licores "Hora Segura” y los muertos por choques de tránsito y atropellos, homicidios y suicidios. La investigación descriptiva en base a los reportes mensuales que realiza la Municipalidad Metropolitana de Lima (LM), Perú de la base de datos del Instituto de Medicina Legal (IML) registrados del 2015 al 2017, con un análisis bivariado y riesgos relativos, caracterizando a los fallecidos por muertes violentas y su relación con sexo, edad y consumo de alcohol. Existe una disminución significativa 
de víctimas alcoholizadas en todas las muertes violentas excepto el suicidio de mujeres, entre el 2015 y 2017. La mayor disminución porcentual en los homicidios de alcoholizados se produjo en las mujeres, significando una reducción del feminicidio. La media de edad se desplaza en frecuencias conforme pasan los años, con excepción del suicidio en mujeres entre el año base (2015) contra el 16 y 17. Las víctimas son mayores en los alcoholizados de LM, coherente con lo publicado por el anuario estadístico de la Policía Nacional, tanto en edad como en sexo. Se concluye que la disminución de muertes violentas y los cambios en edad y sexo en LM debería ser consecuencia de la política pública y los eficaces controles policiales. La "hora segura” tiene evidencias para su aplicación en todo el país. La hora segura es una estrategia eficaz para enfrentar el feminicidio.

Palabras clave: atropellos - choque de tránsito - homicidios - muertes violentas - restricción de horarios de expendio de licores - suicidios

\section{INTRODUCCIÓN}

Los choques de tránsito y atropellos se registraron anualmente en el Perú, entre 74672 a 102 762, en el periodo $2004-2016$, registrándose en el 2016 , unos 89304 en todos los departamentos del Perú, de los cuales 49304 (55\%) ocurrieron en el Departamento de Lima, Perú (INEI, 2018; Perú-MI-PNP, 2018). Estos eventos siguieron una tendencia al afirmar que el incremento del parque automotor correlacionó muy bien con el incremento de estos, particularmente desde 1998 (Loayza et al., 2 008).

En diciembre del 2011 y con el respaldo de un estudio realizado en el Municipio de La Victoria, de Lima Metropolitana en el que se evidenció una disminución de los heridos en riñas y agresiones, homicidios y suicidios relacionada con la disminución de los horarios de expendio de licores, no teniendo efecto en las muertes en choques de tránsito y atropellos, por ser estos mayormente diurnos, con un $25,1 \%$ de las muertes violentas con alcohol en mayor cantidad que $0,5 \mathrm{~g}$ por L de sangre, $\mathrm{y}$ en los varones mayor que en el femenino (Málaga et al., 2012). Se ha promulgado la ordenanza 1568 de restricción de los horarios de expendio de licores, a la que se le dio un plazo de 3 ańos para ser establecida en los 43 municipios que conforman Lima Metropolitana (LM), Perú.

En su aplicación se pueden concebir dos periodos: Primer Periodo diciembre 2011 a diciembre 2014. Segundo periodo enero 2015 a diciembre 2017. Los años base para estos dos periodos, serían el 2010, enero-noviembre 2011(cuando no existía la ordenanza 1568) y el 2015 el ingreso de nuevos alcaldes, también llamado año perdido en la evolución de la hora segura y cambio en el control de la alcoholemia al pasar a tolerancia cero por el Instituto de Medicina Legal (IML). Esto se evidenció en los 13 municipios que no cumplieron con ella (Huánuco, 2019).

Estando en funcionamiento la hora segura, en marzo del 2012, se estableció un Observatorio, el que analizó datos provenientes del IML, relacionados con las muertes violentas (choques de tránsito y atropellos, suicidios y homicidios). Los meses de diciembre del 2011, y enero y febrero del 2012, fueron analizados para la Municipalidad por el primer autor del presente trabajo.

En tal sentido, el objetivo del presente trabajo es determinar la relación entre los Horarios de expendio de licores "Hora Segura" y los muertos por choques de tránsito y atropellos para el periodo 2015-2017, pues el periodo anterior 2011-2014, fue analizado en anterior trabajo (Málaga et al., 2018) en base a la evaluación del efecto del establecimiento de estos horarios en los distritos de LM, Perú.

\section{MATERIAL Y MÉTODOS}

Lima Metropolitana (LM) con 9320.000 habitantes (INEI, 2018) está conformada por 43 municipios, cada cual posee un gobierno municipal, dirigido por un alcalde electo cada cuatro ańos y autónomo en su gestión. La Municipalidad Metropolitana, a través de su concejo metropolitano, aprobó la ordenanza 1568, cuya función principal, es la restricción de horarios de venta de bebidas alcohólicas, estableciéndose que los establecimientos comerciales pueden vender licores desde las 9:00 am 
hasta las 11:00 pm, los restaurantes de domingos a jueves hasta la media noche y los, viernes y sábado, hasta las 3:00 am. Las discotecas, domingos a miércoles hasta la 1:00 am y de jueves a sábados hasta las 3:00 am; y los bares y cantinas, todos los días hasta las 11:00 pm (El Peruano, 2011). Esto de acuerdo con los alcaldes distritales se debería establecer en tres años.

El IML, envía a la ML, una información mensual en Excel relacionada con las muertes violentas, en donde se caracteriza la siguiente data: Número de código, ingreso, hora, historia clínica, sexo, domicilio, edad, grado de instrucción, ocupación, estado civil, clase de accidente: homicidio, suicidio, accidente de tránsito, lugar de ocurrencia, hora del accidente, alcoholemia y procedencia (Málaga, 2009).

Al ser un estudio descriptivo se calcularon variables absolutas y frecuencias relativas y estimación de riesgos relativos y su significancia estadística, utilizando el Programa EPI -Info 7 del CDC (Center for Disease Control and Prevention), para describir la caracterización de los datos de muertos por choques de tránsito y atropellos, homicidios y suicidios, así como para las variables categóricas sexo, edad y alcoholemia. Para el periodo enero 2016-diciembre 2017, usaremos como año base el año 2015, esto por la transición del cambio de autoridades locales en enero del 2015 (alcaldes) y haberse cambiado el criterio de cadáver alcoholizado por el IML, de $0,5 \mathrm{~g}$ de alcohol por $\mathrm{L}$ de sangre a tolerancia cero ese mismo ańo.

\section{Aspectos éticos}

Los autores declaran que se cumplió con toda la normatividad ética nacional e internacional.

\section{RESULTADOS}

Entre el 2015 y el 2017, el Instituto de Medicina legal examinó 1610 cadáveres de muertes violentas con alcohol (choques de tránsito y atropellos, homicidios y suicidios) en LM, 761 de estas eran muertes por choques de tránsito y atropellos, 566 homicidios y 283 suicidios.

Los cadáveres sin alcohol fueron 895 de choques de tránsito y atropellos, 527 homicidios y 287 suicidios para un total de 1709 muertes violentas. En total la morgue en este periodo realizó la necropsia en 3319 cadáveres de muertes violentas.

Entre el 2015 al 2017, hubo una clara tendencia a disminuir en los tres tipos de eventos (Tablas 1 y 2), pues en los tres eventos la disminución es significativa o altamente significativa, con excepción de la comparación en suicidios, para el periodo 2015-2016.

Tabla 1. Comparación de $\mathrm{RR}$ entre hallazgos de alcohol en la sangre ( $\geq 0,01 \mathrm{~g}$ de alcohol por $\mathrm{L}$ de sangre) en cadáveres de muertes violentas. Instituto de Medicina Legal (2015-2016). Ch T= Choques de Tránsito y atropellos.

\begin{tabular}{|c|c|c|c|c|c|c|c|}
\hline Victimas & $\begin{array}{l}\text { Ante } \\
201\end{array}$ & $y \%$ & $\begin{array}{l}\text { Des } \\
201\end{array}$ & F y \% & $\mathrm{RR}$ & $\begin{array}{l}\text { Límites de } \\
\text { Confianza }\end{array}$ & $\mathrm{X}_{1 \mathrm{gl}}^{2}\left(\operatorname{probX}^{2}\right)$ \\
\hline $\mathrm{Ch} \mathrm{T}$ & 326 & 54,5 & 234 & 45,9 & 1,19 & $1,05-1,34$ & $7,86 \quad 0,005$ \\
\hline Suicidios & 114 & 58 & 91 & 44,4 & 1,22 & $0,99-1,49$ & $3,47 \quad 0,06$ \\
\hline Homicidios & 232 & 59 & 161 & 40,1 & 1,26 & $1,09-1,45$ & $10,27 \quad 0,001$ \\
\hline Total & 672 & 58 & 486 & 42,0 & 1,21 & $1,11-1,30$ & 21.67 .0000 \\
\hline
\end{tabular}

Tabla 2. Comparación de RR entre hallazgos de alcohol en la sangre ( $\geq 0,01 \mathrm{~g}$ de alcohol por $\mathrm{L}$ de sangre) en cadáveres de muertes violentas. Instituto de Medicina Legal (2015-2017). Ch T= Choques de Tránsito y atropellos.

\begin{tabular}{|c|c|c|c|c|c|c|c|}
\hline Victimas & $\begin{array}{l}\text { Antes } \\
2015\end{array}$ & y \% & $\begin{array}{l}\text { Desp } \\
2017\end{array}$ & F y \% & $\mathrm{RR}$ & $\begin{array}{l}\text { Límites de } \\
\text { Confianza }\end{array}$ & $\mathrm{X}_{1 \mathrm{gl}}^{2}\left(\right.$ probX $\left.^{2}\right)$ \\
\hline Ch T & 326 & 54,5 & 272 & 36,7 & 1,49 & $1,30-1,70$ & $35,9 \quad 0,0000$ \\
\hline Suicidios & 114 & 58 & 78 & 43 & 1,36 & $1,1-1,67$ & $8,22 \quad 0,003$ \\
\hline Homicidios & 232 & 60 & 173 & 47 & 1,29 & $1,12-1,48$ & $13,270,0003$ \\
\hline Total & 672 & 58 & 457 & 49 & 1,38 & $1,26-1,50$ & $55,130,0000$ \\
\hline
\end{tabular}


En los choques de tránsito y atropellos la reducción de cadáveres alcoholizados se produjo en varones y en mujeres en proporciones similares, $23,4 \%$ para los varones y $25,2 \%$ para las mujeres (Tabla 3 ).

Tabla 3. Comparación de RR entre hallazgos de alcohol en la sangre ( $\geq 0,01 \mathrm{~g}$ de alcohol por L de sangre) en cadáveres de muertos por choques de tránsito, según sexo. Instituto de Medicina Legal (2015-2017).

\begin{tabular}{lccccccc}
\hline Victimas & \multicolumn{2}{c}{$\begin{array}{l}\text { Antes F y \% } \\
\text { 2015 }\end{array}$} & \multicolumn{2}{l}{$\begin{array}{l}\text { Después F y \% } \\
2017\end{array}$} & RR & $\begin{array}{l}\text { Límites de } \\
\text { Confianza }\end{array}$ & $\mathrm{X}_{\text {1gl }}^{2}\left(\right.$ probX $\left.^{2}\right)$ \\
\hline Varones & 259 & 61,7 & 161 & 38,3 & 1,36 & $1,2-1,5$ & $22,20,000002$ \\
Mujeres & 67 & 62,6 & 40 & 37,4 & 1,51 & $1,2-1,9$ & $11,80,0006$ \\
\hline
\end{tabular}

En los homicidios la reducción de cadáveres alcoholizados mujeres y solo $12,4 \%$ en varones (Tabla 4 ). se redujo más en mujeres que en varones; $22,8 \%$ en

Tabla 4. Comparación de RR entre hallazgos de alcohol en la sangre ( $\geq 0,01 \mathrm{~g}$ de alcohol por litro de sangre) en cadáveres de homicidios, según sexo. Instituto de Medicina Legal (2015-2017).

\begin{tabular}{lcccccccc}
\hline Victimas & \multicolumn{2}{l}{$\begin{array}{l}\text { Antes F y \% } \\
\text { 2015 }\end{array}$} & $\begin{array}{l}\text { Después F y \% } \\
2017\end{array}$ & RR & $\begin{array}{l}\text { Límites de } \\
\text { Confianza }\end{array}$ & $\mathrm{X}_{\text {1gl }}^{2}\left(\right.$ probX $\left.^{2}\right)$ \\
\hline Varones & 205 & 56,2 & 160 & 43,8 & 1,23 & $1,04-1,43$ & 6,5 & 0,01 \\
Mujeres & 27 & 61,4 & 17 & 38,6 & 1,45 & $0,99-2,12$ & 2,92 & 0,09 \\
\hline
\end{tabular}

En los suicidios tuvo un efecto de disminución 23\% las muertes con cadáveres alcoholizados (Tabla 5). significativa solo en los varones, quienes redujeron en un

Tabla 5. Comparación de RR entre hallazgos de alcohol en la sangre ( $\geq, 01 \mathrm{~g}$ de alcohol por litro de sangre) en cadáveres de suicidas, según sexo. Instituto de Medicina Legal (2015-2017).

\begin{tabular}{|c|c|c|c|c|c|c|c|c|}
\hline Victimas & $\begin{array}{l}\text { Ant } \\
201\end{array}$ & $\mathrm{y} \%$ & & y\% & $\mathrm{RR}$ & $\begin{array}{l}\text { Límites de } \\
\text { Confianza }\end{array}$ & $\mathrm{X}_{1 \mathrm{gl}}^{2}(\mathrm{I}$ & $\left.\mathrm{bX}^{2}\right)$ \\
\hline Varones & 88 & 61,5 & 55 & 38,5 & 1,45 & $1,05-2,01$ & 5,47 & 0,02 \\
\hline Mujeres & 26 & 53,1 & 23 & 46,9 & 1,25 & $0,84-1,86$ & 0,84 & 0,36 \\
\hline
\end{tabular}

En cuanto a la edad tanto en los choques de tránsito y atropellos y homicidios, de los alcoholizados, esta presenta un incremento de edad en las victimas, en los suicidios estos solo es perceptible entre el 2015 y el 2017 (Figuras 1, 2 y 3).
En los no alcoholizados los perfiles de edad de las víctimas son similares, entre los diferentes años (Figuras 4, 5 y 6). 


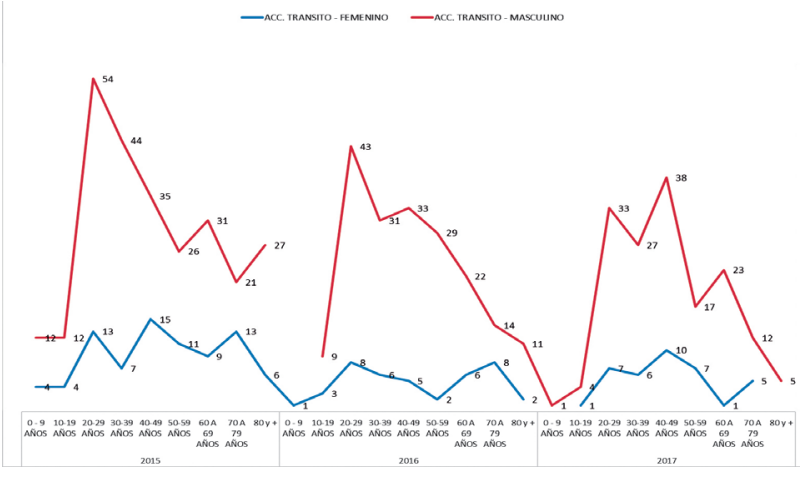

Figura 1. Muertes causadas por choques de tránsito y atropellos relacionados al alcohol según sexo y edad Año 2015 al 2017.

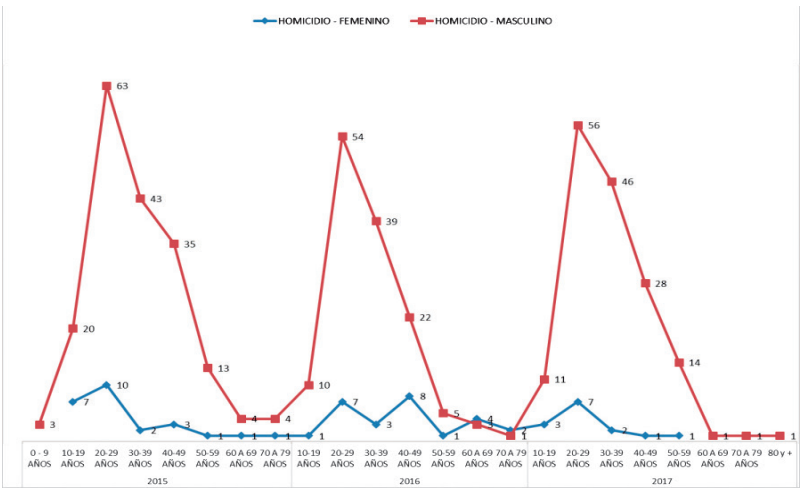

Figura 2. Muertes causadas por homicidios relacionados al alcohol según sexo y edad - Año 2015 al 2017.

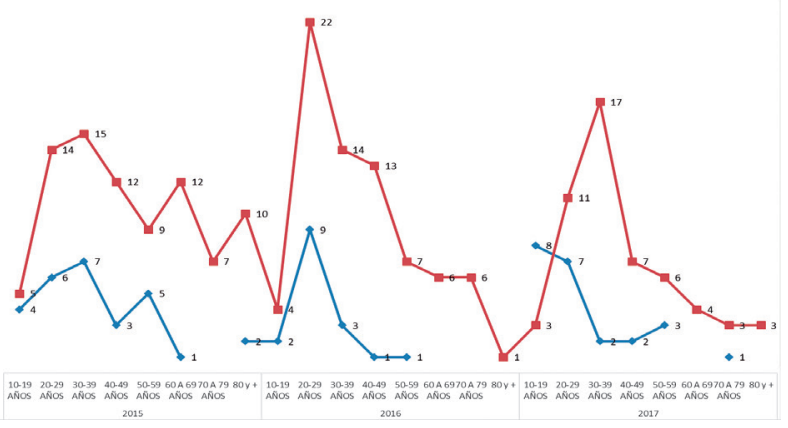

Figura 3. Muertes causadas por suicidios relacionados al alcohol según sexo y edad - Año 2015 al 2017.

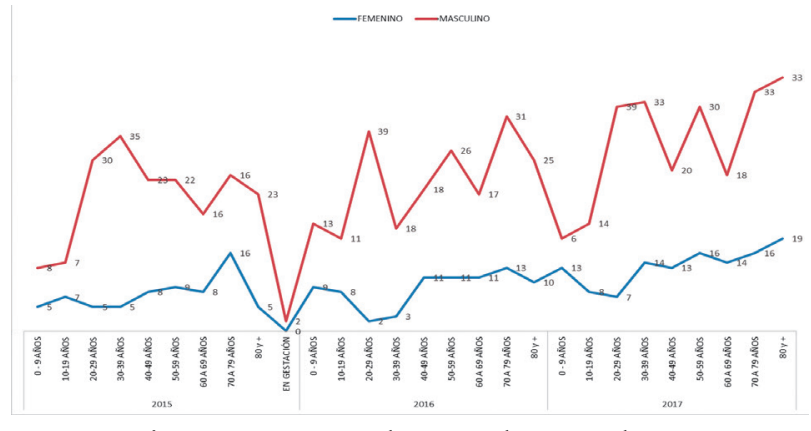

Figura 4. Muertes causadas por choques de tránsito y atropellos en personas no alcoholizadas según sexo y edad - Año 2015 al 2017.

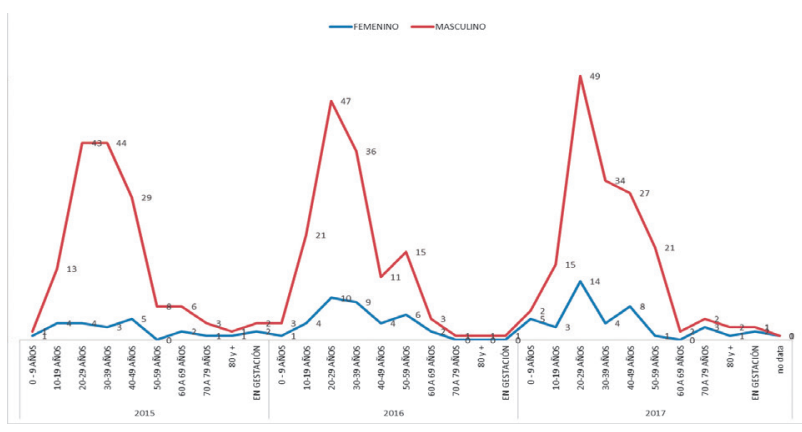

Figura 5. Muertes causadas por homicidios en personas no alcoholizadas según sexo y edad - Año 2015 al 2017.

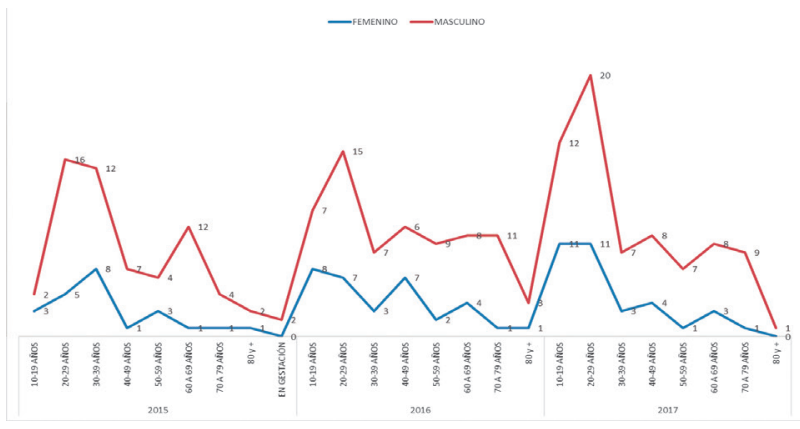

Figura 6. Muertes causadas por suicidios en personas no alcoholizadas según sexo y edad - Año 2015 al 2017. 


\section{DISCUSIÓN}

Los datos de la Policía Nacional del Perú, del 20042016, corroboran que los Choques de tránsito y los atropellos están correlacionados con el crecimiento del parque automotor hasta el 2014, y evidencian que estos en el Departamento de Lima, tienen un comportamiento diferente al comportamiento nacional y del resto del país, ya que Lima tiene una tendencia lineal, que no refleja los incrementos nacionales (Málaga et al., 2018), por lo que nuestros hallazgos de disminución de las muertes violentas y en especial de las victimas alcoholizadas, debería ser consecuencia de la restricción de horarios de expendio. Esta medida con otros programas que regulen enfatice y penalice a los conductores bajo la influencia del alcohol, mejoren la seguridad en el manejo, mejoren la infraestructura de carreteras y establezcan programas de educación y castigos a los conductores con antecedentes de violaciones de tránsito (Lefio et al., 2018), evidenciado en Brasil que reducen los eventos de tránsito (De Souza \& Mathias, et al., 2018).

El incremento de muertes violentas por choques de tránsito y atropellos ,que no registran alcohol en la sangre nos ha llevado a determinar la necesidad de enfatizar en otras medidas como las de señalización de lugares peligrosos (puntos negros), y mejorando a través de esta ubicación, el control de la velocidad como lo realizado en la provincia del Callao (Perú-MPCGTU, 2013). No obstante no podemos desmayar en el monitoreo de la hora segura, que ha evidenciado una importante disminución de las muertes violentas, en nuestro estudio y en la aplicación de esta política pública en otras ciudades como: Bogotá, Cali y Medellín en Colombia, Asunción en Paraguay y Diadema en Sao Paulo, Brasil (MA, 2004; Málaga, 2005; Huanuco, 2019; Maldonado, 2008; Dualibi et al., 2007; De Souza \& Mathias, 2018; Málaga et al., 2018). En Cali, Colombia, también se evidenció una correlación entre el número de horas de expendio de licores y la tasa de homicidios, lo que también se observó en La Victoria, Lima con las agresiones (Sánchez et al., 2011; Málaga et al., 2012).

Esta hora segura en LM, ha evidenciado que ha sido capaz de reducir las muertes violentas de alcoholizados en horas de la madrugada, todos los días de semana con excepción de los viernes, principalmente de varones jóvenes (Málaga et al., 2018).

Las victimas del sexo masculino fueron más afectadas en Manizales, Colombia antes de la intervención (Arias \& Sáenz, 1999). Situación similar se presentó en la ciudad de Arequipa, Perú con un prevalencia de $31,6 \%$ en varones y 11,8\% en Mujeres con respecto al consumo de alcohol en jóvenes (Huarachi, 2018). En nuestro caso, la disminución de la victimas alcoholizadas fue similar tanto en varones como en mujeres en los choques de tránsito y atropellos, y homicidios, estando siempre más afectados los varones. La mayor disminución porcentual en los homicidios de alcoholizados se produjo en las mujeres, lo que significaría una reducción del feminicidio.

En los suicidios los cadáveres disminuyeron solo en los varones alcoholizados. Esto es confirmado por las estadísticas nacionales de la Policía, pues el departamento de Lima, posee un porcentaje menor de defunciones en mujeres por choques de tránsito y atropellos que el resto del país (Perú-MI-PNP, 2018).

Con relación a la edad de las víctimas, el desplazamiento de edad se evidencio en Cali en los homicidios (Espitia $e t$ al., 2002), lo que también es confirmado por la policía, en su anuario estadístico, al evidenciar que la edad de los muertos en Lima en choques de tránsito y atropellos es mayor que en el resto del país (Perú-MI-PNP, 2018).

El horario de expendio de licores "Hora Segura" tiene relación con la disminución de los muertos por choques de tránsito y atropellos, homicidios y suicidios en sus víctimas alcoholizadas. El hecho que la disminución es más marcada en los alcoholizados que en los no alcoholizados, definiría, un indicador de eficacia del programa. La mayor disminución porcentual de homicidios en alcoholizados se produjo en las mujeres, lo que significaría que la hora segura es una buena estrategia para enfrentar el feminicidio. Esta disminución es evidente en varones y mujeres con excepción de las mujeres en caso de suicidios.

La edad de las victimas alcoholizadas, en choques de tránsito y atropellos y homicidios es más tardía, conforme ocurre la disminución de estos, en los no alcoholizados no se observa esta diferencia. Los choques de tránsito y atropellos de los no alcoholizados, están en incremento, sugiriéndonos la necesidad de incidir en el control otros factores de riesgo como el control de velocidad y el mejoramiento de la señalización en los puntos negros.

\section{REFERENCIAS BIBLIOGRÁFICAS}

Arias, B \& Sáenz, X. 2018. Evaluación de la ley zanahoria mayo 97-99 http://telesalud.ucaldas.edu.co/ telesalud/facultad/Documentos/Promocion/ Vol\%206/Evaluacion \% 20de\% 20la \% 20 Ley\%20Z.pdf consultado el 28 de agosto 2018 
De Souza, A. \& Mathias, T. 2018 Impacto do código de trânsito Brasileiro e da Lei Seca na mortalidade por acidentes de Trânsito. Cadernos de Saúde Pública, 34: e00122117.

Duailibi, S.P.; Pinsky, W.; Laranjeira, I. \& Raw, M.R. 2007 The effect of restricting opening hours on Alcohol-related violence. American Journal of Public Health, 97: 2276-2280.

Espitia, A.A. \& Espinoza-Gutiérrez, M.I. 2002. Muertes por homicidio, ocurridas en el año 2002 CISALVA, Cali, Colombia en http:// vigilesiones.univalle.edu.co/informes/anual/ homicidios/homic_2002.pdf consultado el 26 de enero 2018 .

Huánuco, M. 2019. Restricción de horas en el consumo, expendio de bebidas alcohólicas y mortalidad por accidentes de tránsito en Lima Metropolitana 2010 - 2015. Tesis Maestría. Facultad Enfermería UNC, Huancayo - Perú.

Huarachi, J. 2018 Tipos de consumo de alcohol en estudiantes de un centro pre-universitario de Arequipa en abril del 2018. Tesis Médico Cirujano. UNSA (Universidad Nacional de San Agustín). Perú.

Lefio, A.; Bachelet, V.C.; Jiménez-Paneque, R.; Gomolán, P. \& Rivas, K. 2018 A systematic review of the effectiveness of interventions to reduce motor vehicle crashes and their injuries among the general and working populations. Revista Panamericana de Salud Pública, 42: e60.

Loayza, M.; Rojas, C., Cisneros, G.; Salvador, M. \& Boloarte, J. 2008. Epidemiological characteristics of road traffic accidents in Perú, 2000 - 2006. $9^{a}$ Conferencia Mundial sobre prevención de lesiones y promoción de la seguridad; 2008 marzo 15-18, Mérida, México.

Málaga, H. 2005. Politicas públicas saludables y libertades básicas. In: Málaga, H. (Ed). Salud Pública Enfoque Bioético. Caracas, Venezuela: DISINLIMED. p. 147-57.

Málaga, H. 2009. Restricción de horarios de expendio de Bebidas Alcohólicas (Ordenanza Municipal 0087) y su posible relación con la violencia en el periodo 2005-2008 en el Municipio de La Victoria, Lima,
Perú. Tesis Doctor en Salud Pública. Universidad Peruana Cayetano Heredia. Lima, Perú.

Malaga, H.; Gonzalez, M.; Huaco, C. \& Sotelo,M. 2012. The relation between the number of hours that authorize the sale of alcoholic beverages and violence. Health Promotion Perspectives, 2: 60-71.

Malaga, H.; Palza, G.; Huánuco. M.; Ñañez, J. \& Ronceros, J. 2018. The restriction of hours of sales of liquors: Lima 2010-17: Traffic accidents (TA) and other violent deaths. Journal of Alcoholism and Drug Dependence, 6: 1000306.

Maldonado, M. 2008 Seguridad Vial, un desafio para la Salud Pública. In: Externas DdVyLdC. (Ed). Asunción, Paraguay: Artes 13.

MA (Municipalidad de Asunción). 2004. Plan Municipal y Proyecto de Prevención de la Violencia Promoción de la Convivencia Ciudadana. In: Salud-DGAS. Dd, Editor. Asunción, Paraguay. p. 51.

Perú, MI (Ministerio del Interior), PNP (Policía Nacional del Perú). 2018. Anuario Estadístico 2017. División de Estadística.

Perú Rd. El Peruano. 2011. Establecen el nuevo régimen municipal que regula la comercialización, consumo y publicidad de bebidas alcohólicas de toda graduación. Ordenanza No 1568, 2011, 3 de diciembre 2011; Sect. 453445-348

Perú-MPCGTU (Municipalidad Provincial del Callao Gerencia General de Transporte Urbano). 2013. Identificación de puntos negros de accidentes de tránsito en el Callao.

Perú Rd. INEI. 2018. Instituto Nacional de Estadistica e Informática. INEI. Lima.

Sánchez, A.; Villaveces, A.; Krafty, R.; Park, T.; Weiss, H.; Fabio, A.; Puyana, J.C. \& Gutiérrez, M.I. 2011 Policies for alcohol restriction and their association with interpersonal violence: a timeseries analysis of homicides in Cali, Colombia. International Journal of Epidemiology, 40: 1037-1046.

Received October 29, 2019.

Accepted December 2, 2019. 